\title{
Impact of Guidelines on the Diffusion of Medical Technology: A Case Study of Cardiac Resynchronization Therapy in the UK
}

\author{
Rucha Vadia $^{1,2}$ (1) $\cdot$ Tom Stargardt $^{1}$
}

Accepted: 2 September 2020 / Published online: 24 September 2020

(c) The Author(s) 2020

\begin{abstract}
Introduction Research on clinical practice guidelines as a determinant of the diffusion of medical technology remains sparse. We aim to evaluate the impact of guidelines on the awareness of medical technology, as a proxy of its use, with the example of cardiac resynchronization therapy (CRT) in the United Kingdom (UK).

Methods We measured clinician awareness based on Google searches performed for CRT that corresponded with actual CRT implant numbers provided by the European Heart Rhythm Association (EHRA). We identified the guideline recommendations published by the National Institute of Health and Care Excellence (NICE) within the UK, the European Society of Cardiology (ESC) at the European level, and the American College of Cardiology Foundation/American Heart Association in the United States (US). We specified a dynamic moving average model, with Google searches as the dependent variable and guideline changes as the independent variables.

Results One guideline change published by NICE in 2007 and two changes released by the US guidelines in 2005 and 2012 were significantly correlated with the Google searches $(p=0.08, p=0.02$, and $p=0.02$, respectively). Guideline changes by the ESC had no significant impact. Changes recommending CRT in place of a conventional pacemaker, in patients with atrial fibrillation, and restricting CRT due to contraindication, remained universally uninfluential.

Conclusion The factors associated with a lack of awareness (as a proxy for technology diffusion) in our case study were: a lack of strong clinical evidence that resulted in the moderate strength of a recommendation, a lack of recognition of any externally published recommendation by NICE, and the frequent release of guidelines with minor changes targeting small patient groups. At least in our case, in the absence of NICE guidelines, the US guidelines received more attention than their non-UK European counterparts, even if the former were released after the latter.
\end{abstract}

Electronic supplementary material The online version of this article (https://doi.org/10.1007/s40258-020-00610-8) contains supplementary material, which is available to authorized users.

Rucha Vadia

rucha.vadia@abbott.com; rucha.vadia@uni-hamburg.de

1 Hamburg Center for Health Economics, University of Hamburg, Esplanade 36, 20354 Hamburg, Germany

2 Abbott, Health Economics \& Reimbursement, Da Vincilaan 11, 1935 Zaventem, Belgium

\section{Key Points for Decision Makers}

Awareness-and thus presumably use of - CRT in the UK is associated with one NICE guideline from 2007 and two US guidelines from 2005 and 2012.

Strong clinical evidence, reflected by the strength of guideline recommendation, seems to be important for clinician response to the guideline.

When national guidelines were absent, the US guidelines rather than the European ones were correlated with the awareness of CRT in the UK, even if released later. 


\section{Introduction}

Diffusion of innovations has been defined as "the process by which an innovation is communicated through certain channels over time among the members of a social system" [1]. Research on the diffusion of medical technology can be traced back to as early as the 1950s and work such as the Columbia University Drug Study in the United States [2]. Study of the determinants of the diffusion of innovation in healthcare services is spread across a wide range of disciplines, including sociology, technology, sociopolitics, development and organizational research, among other areas [3, 4]. We identified some major determinants of medical technology diffusion from empirical studies, which were classified using the model proposed by Meyer and Goes: (1) innovation attributes, (2) contextual attributes, and (3) innovation-decision attributes of clinicians/ providers [5, 6].

First, in relation to innovation attributes, several studies have discussed how technological attributes that focus on unmet needs, additional benefits, and usability and interoperability act as positive determinants of technology diffusion $[7,8]$. Second, the context of a country, in terms of financial, economic, and regulatory policies, also influences the diffusion of medical technology. For example, comparative studies among European and other OECD countries have shown that gross domestic product (GDP) and healthcare expenditure per capita positively correlate with the adoption of new medical technologies [9-13]. In addition, it has been shown that while wealthier countries adopt new technologies earlier, access to these technologies becomes less dependent on income over the long term [10]. Funding and procurement policies, reimbursement strategies, and types of reimbursement - either at hospital or clinician level-have been demonstrated to be determinants of diffusion [14-21]. Studies in the United States (US) hospitals have analyzed how changes in reimbursement policies lead to different rates of technology diffusion; for example, prospective reimbursement leading to lower diffusion rates [17, 19]. Moreover, an analysis of diagnosis-related-group reimbursement in the Italian NHS found the reimbursement mechanism more important for technology diffusion than the magnitude of reimbursement [22].

Third, in relation to studies on the innovation-decision attributes of clinicians/providers, to which we have also contributed, multiple decision-making systems (medical, managerial, and strategic) of the providers have been shown to be involved in technology adoption decisions [23]. Clinicians' perceptions of the additional benefits of technology, as well as externality, affect the adoption rate $[8,24]$. Learning effects and knowledge about how to utilize a given technology have also been shown to be crucial determinants at the organization level $[25,26]$.
One such form of knowledge, evidence-based medicine, has an evolving influence on clinicians' decisions concerning technology uptake [3, 27]. Clinical practice guidelines are a common element of evidence-based medicine, intended to reduce uncertainty about optimal decision making by sharing recommendations based on collective research evidence as well as expert opinions [28]. However, change in technology uptake in response to guidelines is heterogeneous $[3,29]$. It has been suggested that impact of clinical guidelines with respect to technology diffusion, and ultimately technology utilization, should be further explored [30]. This case study intends to take a first step in understanding the role of guidelines in the diffusion of medical technology, aiming to evaluate: (1) whether the publication of guidelines affects the awareness of medical technology and (2) whether a guideline released by a national, European, or US body affects awareness. Our case study investigated one specific example of technology diffusion: cardiac resynchronization therapy for the treatment of heart failure (HF) in the United Kingdom (UK).

\section{Background}

HF starts with an injury to cardiac tissue that is compensated by the heart using various mechanisms over the short term. In the long term, these compensatory mechanisms give rise to one or more symptoms that characterize the syndrome. One of these long-term symptoms results in ventricular dyssynchrony, an imbalance in the pumping of blood either between the left and the right ventricles or in the left ventricle only [31]. Cardiac resynchronization therapy (CRT) involves implanting a device that electrically targets ventricular dyssynchrony with its more advanced composition than the standard pacemaker system. With the help of an additional lead, CRT aims to synchronize left-ventricular movement and thus its blood-pumping activity [32]. It has been shown that CRT's pacing mechanism reduces morbidity and mortality and improves functional capacity in HF patients $[33,34]$.

Nevertheless, patient response to CRT differs widely, with approximately $30 \%$ of patients inexplicably found to be non-responders. Apart from some overlapping clinical predictors, a single root cause of this non-responsiveness has not yet been identified $[32,35]$. Thus, the diverse mechanisms behind non-responsiveness to CRT, in addition to already diverse HF symptoms, makes the implantation decision at the clinician level crucial. This, in turn, makes an analysis of clinicians' responses to the release of guidelines interesting and valuable.

In addition, since the first randomized controlled trial in 1995, the patient selection criteria for CRT implantation have been modified in amendments to guidelines [32, 36]. 
One of the aims of the clinical practice guidelines for CRT has been to optimize the patient selection criteria to assist clinicians in the absence of certainty about CRT response.

\section{Methods}

We chose the UK for our analysis because: (1) it has its own set of national guidelines that are published and revised on a regular basis, mainly by the National Institute for Health and Care Excellence (NICE), (2) it participates in and is also directed by task forces of the European Society of Cardiology (ESC) at the European level, and (3) because of the lack of a language barrier, it is likely to pay attention to guidelines released in the US by the American College of Cardiology Foundation (ACCF), the American Heart Association (AHA), and the Heart Rhythm Society (HRS).

\subsection{Data}

To approximate the use of CRT, we measured awareness about it by analyzing Google searches for CRT over time. Google Trends, a website established by Google, provides data about searches performed in Google's search engine. We chose this approach because trends in search volumes from Google in the UK and the actual figures for CRT implantations in the UK per million inhabitants, which are available from the European Heart Rhythm Association (EHRA), substantially correspond (see Fig. 1) [37]. We found a cross-correlation coefficient of 0.91 between two time series [38]. In addition, there is evidence that clinicians' use of knowledge found online is related to direct patient care $[39,40]$ and that the Google search platform is widely used to gain access to the online medical literature [41].
Google Trends provides an unbiased sample of Google searches as a percentage of total anonymized Google searches available for a particular search term in a region. We selected the UK as our geographical region, which in this time series covered Wales, England, and Scotland. Each data point of the query from the sample was divided by the total search volume in the region and then scaled from 0 to 100 . The lowest number of searches was scored as 0 , while the highest number was scored as 100. Duplicate searches from the same person over a short period of time were removed [42]. For our query, we used the search term "cardiac resynchronization therapy" as a Google topic that covers all CRT-related terms, including different forms of the technology, such as CRT-P, which stands for CRTPacemaker and CRT-D, which stands for CRT-Defibrillator; synonyms of the term, such as "resynchronization therapy" or "bi-ventricular pacemaker"; spelling variations such as "resynchronization"; and acronyms. Google topic also includes search queries made in foreign languages if present in the search database. In this manner, we produced a monthly time trend of Google searches from 1 January 2004 through 30 June 2018.

\subsection{CRT Guideline Changes}

We examined documents released by bodies in the UK, Europe and the US to capture any guideline changes. For the UK, we identified technology appraisals published by NICE, which are valid for England and Wales. Each of the NICE guidelines in our analysis was also appraised and validated by NHS Quality Improvement Scotland for implementation there $[43,44]$. At the European level, we identified guidelines for HF, as well as guidelines for cardiac pacing and CRT, released by ESC task forces. For the US, we identified guidelines on HF and device-based therapy for cardiac
Fig. 1 Search scale for "cardiac resynchronization therapy" topic by Google Trends and number of CRT implantations per million inhabitants by EHRA. CRT cardiac resynchronization therapy, EHRA European Heart Rhythm Association

\section{United Kingdom}

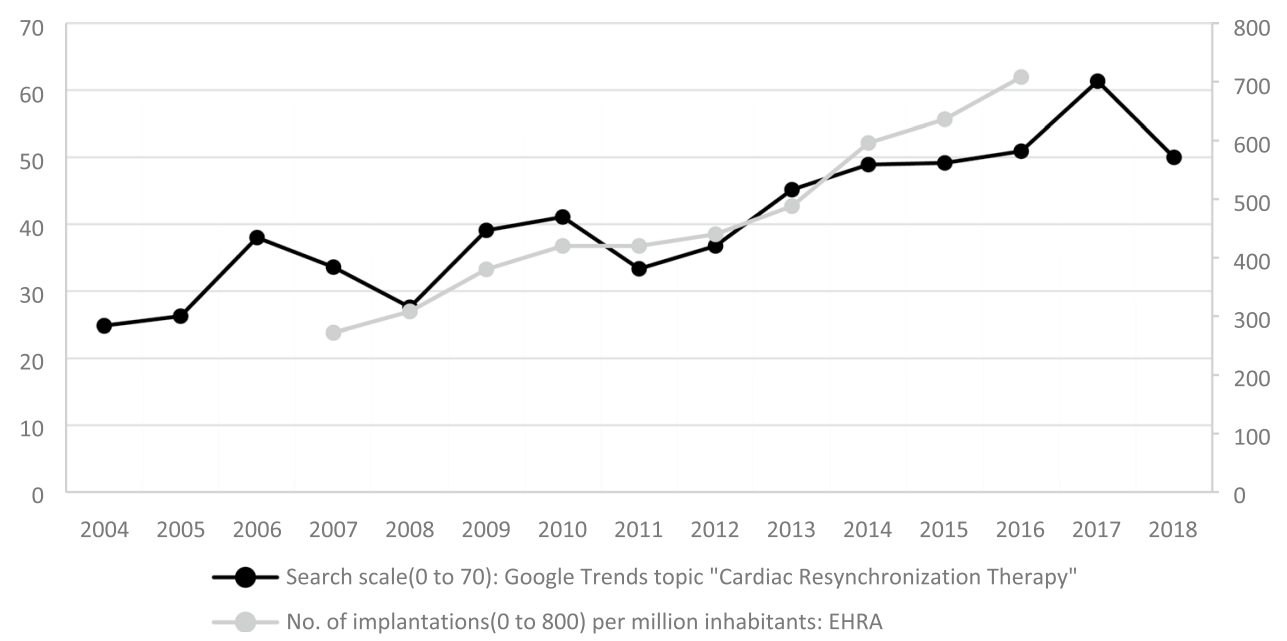


rhythm abnormalities, released by the ACCF/AHA/HRS or ACC/AHA.

We found major changes in patient selection criteria over time, as described by Boriani et al. [53], and grouped these into three main categories, adding another category of changes, which concerned restrictions made to selection criteria. Table 1 describes the categories, along with the degree of strength of recommendation, and the levels of clinical evidence supplied by the respective guideline bodies.

(1) Initial indication: patients with moderate-to-severe heart failure

CRT was initially indicated in patients with sinus rhythm (regular heart rhythm) but with HF classified as moderateto-severe according to the New York Heart Association (NYHA) functional classification system (Classes III-IV). The ESC recommended CRT for this group in 2005 [45]. The ACC/AHA in the US already recommended it for this group in 2002 but the strength of recommendation increased to Class I in 2005 (level A evidence, i.e. strongly recommended) [46, 47]. In the UK, however, there was no CRT implant recommendation until 2007, when NICE published its technology appraisal, TA120 [48].

(2) Extended indications: patients not initially indicated for CRT as primary treatment

Another wave of recommendations that appeared over time broadened indications to patients with additional HF symptoms that were not intended to be primarily treated by CRT. As all of these recommendations diverged from the indication of CRT as the primary treatment for left-ventricular conduction delay, we combined them into one category, subsequently defining each type as a sub-category.

(a) CRT as an alternative to a conventional pacemaker

The ESC guideline of 2007 and the ACC/AHA/HRS guideline of 2008 recommended this change [49, 50].

(b) Patients with atrial fibrillation (AF)

The ACC/AHA/HRS guidelines of 2008 and the ESC guideline of 2010 recommended this extension of CRT use for patients with atrial fibrillation [50,51].

(c) Upgrading implanted pacemaker to CRT

The ESC guideline of 2007 and the ACCF/AHA/HRS guideline of 2012 recommended upgrading an already implanted pacemaker system to CRT in 2007 [49, 52].

(3) Inclusion of patients with asymptomatic-to-mild heart failure

Eventually, the recommendation for patients with sinus rhythm HF was extended to patients with asymptomaticto-mild HF (NYHA functional classes I-II, respectively) [53]. The ESC recommended this extension in 2010, while the ACCF/AHA/HRS recommended it in 2012 [51, 52]. The NICE technology appraisal, TA314, recommended this change in 2014 [54].

Table 1 CRT guideline changes

\begin{tabular}{|c|c|c|c|c|}
\hline Change & Variable name & Publishing body & Date of publication & $\begin{array}{l}\text { Type of recommen- } \\
\text { dation and level of } \\
\text { evidence }\end{array}$ \\
\hline \multirow[t]{3}{*}{ (1) Initial indication of moderate to severe-risk patients } & EU_2005 & ESC & May, 2005 & Class IA \\
\hline & US_2005 & ACC/AHA & September, 2005 & Class IA \\
\hline & NICE_2007 & NICE & June, 2007 & Recommended \\
\hline \multirow{2}{*}{$\begin{array}{l}\text { (2a) Extended indications-patients not initially indicated for } \\
\text { CRT as primary treatment, sub-category: alternative to } \\
\text { pacemaker }\end{array}$} & EU_2007 & ESC-EHRA & September, 2007 & Class II C \\
\hline & US_2008 & ACC/AHA/HRS & May, 2008 & Class II C \\
\hline \multirow{2}{*}{$\begin{array}{l}\text { (2b) Extended indications-patients not initially indicated for } \\
\text { CRT as primary treatment, sub-category: patients with AF }\end{array}$} & US_2008 & ACC/AHA/HRS & May, 2008 & Class II B \\
\hline & EU_2010 & ESC & August, 2010 & Class IIa C/Class IIa B \\
\hline \multirow{2}{*}{$\begin{array}{l}\text { (2c) Extended indications-patients not initially indicated for } \\
\text { CRT as primary treatment, sub-category: upgrade implanted }\end{array}$} & EU_2007 & ESC-EHRA & September, 2007 & Class II C \\
\hline & US_2012 & ACCF/AHA/HRS & September, 2012 & Class IIa B \\
\hline \multirow{3}{*}{$\begin{array}{l}\text { (3) Inclusion of patients with asymptomatic to mild heart } \\
\text { failure }\end{array}$} & EU_2010 & ESC & August, 2010 & Class I A \\
\hline & US_2012 & ACCF/AHA/HRS & September, 2012 & Class I A \\
\hline & NICE_2014 & NICE & June, 2014 & Recommended \\
\hline \multirow[t]{2}{*}{ (4) Restriction to the use by contraindication } & EU_2013 & ESC-EHRA & June, 2013 & Classs III B \\
\hline & US_2013 & ACCF/AHA & October, 2013 & Class III B/Class III C \\
\hline
\end{tabular}

Class I: (therapy) is recommended, Class IIa: (therapy) should be considered, Class IIb: (therapy) may be considered, Class III: (therapy) is not recommended, Level of Evidence A: data derived from multiple randomised controlled trials or meta-analyses, Level of Evidence B: data derived from a single randomised controlled trial or large non-randomised studies, Level of Evidence C: Consensus of opinion of the experts and/or small studies, retrospective studies, registries

ACC American College of Cardiology, ACCF American College of Cardiology Foundation, AF atrial fibrillation, AHA American Heart Association, CRT cardiac resynchronization therapy, EHRA European Heart Rhythm Association, ESC European Society of Cardiology, HRS Heart Rhythm Society, NICE National Institute for Health and Care Excellence 
(4) Restrictions on the use of CRT due to contraindication in patients with certain conditions

One of the changes in recent guidelines explicitly contraindicates CRT for patients with QRS - an imaging biomarker to understand degree of electrical dyssynchrony in the heart $-<120$, thus restricting the selection of patients for CRT [31]. Both of the guideline bodies, the ACCF/AHA and the ESC, adopted this recommendation for all patients in 2013 [55, 56].

We did not include ESC recommendations released in 2008,2012 , or 2016 because they did not make changes with respect to any of the four categories defined above (compared to the preceding recommendations) and only included minor changes, such as a shift in the class of recommendation, the explicit definition of non-left bundle branch block (LBBB) sub-characteristics, and QRS contraindication extended from existing QRS-120 to QRS-130, respectively. Similarly, we did not include one US-guideline update released in 2009, as it served only as a summary of previous recommendations, rather than providing new or modified recommendations [53].

\subsection{Statistical Analysis}

We estimated an auto regressive integrated moving average (ARIMA) model with monthly Google searches as the dependent variable and variables reflecting changes to the guidelines as independent. First, in order to understand the nature of the Google search time series, we performed various tests [57]. The Dickey-Fuller test $(p=0.001)$, the Augmented Dickey-Fuller test $(p<0.0001)$, the Phillips Perron test $(p<0.0001)$, and the KPSS test $(p<0.0001)$ all rejected the evidence of a unit root, thus confirming that our time series was stationary [58-62]. Second, to check the autoregressive (AR) and/or moving-average (MA) nature of the time series, we first plotted the autocorrelation function (ACF) and the partial-autocorrelation function (PACF) graphs of the series (see Supplementary Fig. s1). Observing the PACF graph, the possibility of any AR order was ruled out due to a lack of any significant correlation for any lag. However, a statistically significant first lag in the ACF graph indicated the possibility of MA(1) order. To further validate these findings, we estimated versions of $\mathrm{AR}(1), \mathrm{MA}(1)$, and $\operatorname{ARMA}(1,1)$. However, only the results of the MA(1) model were significant, supporting the earlier graphical representation. In order to select the optimum number of lags in the MA model, we separately estimated versions of it from one up to three lags; Akaike's information criterion (AIC) and the Schwarz information criterion (SBC) were lowest for the one lag model, making the MA(1) model our final model [63].

The final model was thus, $Y_{t}=\mu+\beta X_{t}+\theta \varepsilon_{t-1}+\varepsilon_{t}$, with $Y$ representing monthly Google searches for "cardiac resynchronization therapy", $X$ corresponding to the vector of variables that represent changes in guidelines, and $\varepsilon_{t-1}$ and $\varepsilon_{t}$ being the error terms.

In order to confirm goodness-of-fit for our model, we performed two checks. First, after estimating our final model, a residual autocorrelation check confirmed white noise. Second, a Breusch-Godfrey test for autocorrelation confirmed the absence of autocorrelation in the residuals. We undertook all statistical analyses in SAS version 9.4.

\section{Results}

The yearly average of Google searches was highest in 2017 . The results of our dynamic regression model are shown in Table 2. First, we see a correlation with the US guidelines (US_2005, $p=0.02$ ), which included a recommendation for CRT in patients with moderate-to-severe HF. Second, we see a correlation with the NICE technology appraisal (NICE_2007, $p=0.08$ ) for the same category of guideline change. Third, we see a correlation with the change from the US guidelines (US_2012, $p=0.02$ ) that included patients with asymptomatic to mild HF and the upgrade of implanted devices to CRT in suitable patients. The two other changes in the US guidelines (US_2008, US_2013), the second change in the NICE technology appraisal (NICE_2014), and all of the changes in the European guidelines (EU_2005, EU_2007, EU_2010,EU_2013) did not have a significant impact on the Google searches.

\section{Discussion}

Overall, we observed that the Google searches (and thus presumably awareness as a proxy for diffusion) correlated with some of the changes to the guideline recommendations

Table 2 Results

\begin{tabular}{lcccc}
\hline Variable & Estimate & Standard error & $t$ value & $\operatorname{Pr}>|t|$ \\
\hline MU & 25.22027 & 4.73603 & 5.33 & $<.0001$ \\
MA1,1 & 0.14489 & 0.07886 & 1.84 & 0.0662 \\
EU_2005 & -16.2629 & 10.63643 & -1.53 & 0.1263 \\
US_2005 & 23.7377 & 10.30028 & 2.3 & 0.0212 \\
NICE_2007 & 20.53973 & 11.82154 & 1.74 & 0.0823 \\
EU_2007 & -19.4213 & 13.02267 & -1.49 & 0.1359 \\
US_2008 & 1.84572 & 7.45558 & 0.25 & 0.8045 \\
EU_2010 & -2.11015 & 5.08023 & -0.42 & 0.6779 \\
US_2012 & 15.68682 & 7.18571 & 2.18 & 0.0290 \\
NICE_2014 & 2.84709 & 7.06101 & 0.4 & 0.6868 \\
EU_2013 & -4.72116 & 11.40467 & -0.41 & 0.6789 \\
US_2013 & 4.67918 & 11.64555 & 0.4 & 0.6878 \\
\hline
\end{tabular}


made at the national and US levels. We observed no such correlation, however, with the changes recommended at the European level. We also saw that the changes that correlated with an increase in Google searches were those that aimed to expand the patient groups with sinus rhythm and left ventricular/intraventricular HF symptoms (i.e., categories 1 and 3 in Table 1). In addition, these recommendations were at the highest strength as they were supported by level A clinical evidence (i.e., high-quality randomized controlled trials or meta-analysis were available).

In contrast, the changes that were aimed at diverse patient groups, such as for AF rhythm disorder and right-ventricular pacing [categories 2(a) and 2(b) in Table 1], consistently did not correlate with the Google searches. They also had a moderate strength of recommendation, with level B or level C clinical evidence (i.e., moderate quality clinical studies or experts' opinion available, see Table 1).

The significant impact of the recommendation of the ACC/AHA released in 2005 (US_2005) is unsurprising given that there was no national guideline for CRT at that time. At the same time, there was an unmet need to treat intraventricular conduction delay in $30-50 \%$ of HF patients globally $[64,65]$. Thus, the strong recommendation in the US guideline appears to have made CRT acceptable, at least to early adopters. The significant impact of the same recommendation in the NICE guideline published in 2007 (NICE_2007) is similarly unsurprising given that this was the first recommendation for CRT at the national level.

We observed another significant correlation with Google searches after the release of the ACCF/AHA/HRS guidelines in the US in 2012 (US_2012). This guideline mainly consisted of two changes: (1) the inclusion of patients with asymptomatic-to-mild HF (Category 3 in Table 1) and (2) a recommendation to upgrade implanted devices to CRT in suitable patients [Category 2(c) in Table 1]. The correlation of the first of these changes seems to make sense, given that there was no such recommendation for this patient group previously, while at the same time some clinical evidence had already started showing the benefits of CRT in this group [35, 66]. It was also well reflected in the strength of recommendation (Class I A).

For the second of these changes, the significance possibly indicates a response, through a switch to more precise technology in patients who originally needed treatment for left or intraventricular conduction delay and had been given the standard pacing technologies available in the absence of the bi-ventricular pacing of CRT. This recommendation had only a moderate strength. An observation of the UK participant centers in the recent European CRT Survey II reveals a substantial sufficient percentage of patients associated with each of these changes, although the percentage is higher in the patient group corresponding to Category 3 than to Category 2(c). Approximately 55\% of all CRT implantation patients had asymptomatic-to-mild HF symptoms, while approximately $25 \%$ of all CRT implantation patients were candidates for the upgrade to CRT [67].

The changes that remained universally uninfluential in our analysis were those recommending CRT in place of a conventional pacemaker [Category 2(a) in Table 1], CRT in patients with atrial fibrillation rhythm disorder [Category 2(b) in Table 1], and restrictions due to contraindication of CRT (Category 4 in Table 1). CRT in place of a conventional pacemaker was only recommended on the basis of expert opinion (i.e. level C clinical evidence) until more recently, in 2013, when the BLOCK-HF trial provided strong evidence [68]. The CRT recommendation in patients with atrial fibrillation was also based on observational studies with level B or level C clinical evidence [69]. Thus, a lack of level A evidence at the time of guideline release is possibly one of the underlying reasons for the consistent non-reflection of the changes. In addition, these recommendations were part of ongoing clinical debates and a lack of response to these changes is thus possibly an indication of the varied consensus in clinical practice. As there has been no response from the UK national guideline bodies to accommodate these changes, no acknowledgement of such changes by the national guideline publishing body is another possible underlying reason for the lack of effect.

Additionally, we expected the change in Category 4 in our analysis (i.e., restrictions due to contraindication) to be significantly associated with awareness. However, it is possible that clinicians were already aware and had already reduced the use of CRT in the particular patient population that was restricted by this contraindication. Thus, an already existing awareness in clinical practice is a third possible underlying reason for non-reflection of the guideline change by Google searches.

The bodies releasing the guidelines also appeared to play an important role in awareness and, presumably, use of CRT. It appears that the US guidelines had an impact if they were the first recommendation of their kind (e.g., the initial recommendation in 2005 and the key patient-group extensions in 2012). However, the consistent non-significant association between Google searches and changes released at the European level is surprising at first glance, given the closer geographical proximity and the UK's membership of the ESC. One potential explanation for this could be the magnitude and frequency of the changes. The guidelines at the European level, released by various task forces of the ESC, are generally more numerous and more frequent, comprising minor changes on each occasion. Moreover, recommendations by different ESC task forces sometimes vary for the same indications. For example, upgrading to CRT from already implanted pacemakers/ICDs had been recommended by the ESC guideline for cardiac pacing and cardiac resynchronization therapy since 2007 , while it remained 
absent from the 2010 focused update of the ESC guideline on device therapy in HF. It again reappeared with a higher strength of recommendation in the ESC guideline on cardiac pacing and cardiac resynchronization therapy in 2013. Both factors may lead to the heterogeneous dissemination of clinical information, as well as variation in consensus, which ultimately may reflect variation in real-world clinical practice.

Several studies have analysed the diffusion of clinical practice guidelines themselves in healthcare services research. Such studies range in therapeutic focus from public health programs, such as smoking cessation, to chronic diseases, such as stroke, chronic kidney disease, or diabetes, and to infectious diseases. They are also geographically widespread, including the US, Canada, the Netherlands, and France [70-76]. However, most of these studies primarily evaluated the determinants of guideline diffusion among healthcare professionals, rather than considering the guidelines as one of the determinants of technology diffusion, as in our case. Thus, our results are of limited comparability to these studies.

Moreover, some observational studies have analyzed the utilization of CRT devices in various patient groups [77-81] but few have connected the utilization patterns with changes in guidelines in a wider setting [82]. One study has observed the evolution of guidelines as an underlying reason for increased pharmaceutical utilization in the US [83]. To the best of our knowledge, our study is the first to take the distinct approach of analyzing Google search data to understand the diffusion of CRT and major changes in guidelines as the independent determinant. An international comparison of CRT guidelines identified minor inconsistencies in CRT recommendations in certain patient groups across the guidelines worldwide [84]. These groups included patients with AF, patients suitable for right-ventricular pacing, and patients with specific QRS duration. These groups corresponded with categories 2(a), 2(b), and 4 in our study, which remained universally uninfluential. Our results thus reflect these underlying inconsistencies and the lack of consensus in the global clinical debate on the use of CRT.

Our study has a number of important limitations. First, we used search data provided by Google Trends. Although we established a correlation between search data and CRT use, it can only serve as a proxy for actual use because the increase in searches may not be attributed solely to clinicians but also can be reflected due to the behavior of the public. Second, monthly data for CRT use were unavailable to evaluate the correlation between search data and CRT use further at that level. Third, we only focused on search queries made in the UK. Thus, our results may differ from those found in other European countries, particularly if access to the US guidelines differs because of the language barrier. Fourth, we did not include the guidelines released in Canada, Australia, or New Zealand, primarily focusing on the effects of US-based external guideline developing bodies in the UK setting. Fifth, the appraisal of the NICE guidelines by NHS Quality Improvement Scotland may have delayed the response in Scotland, resulting in heterogeneity. Sixth, although the impact of a specific change on one of the many different CRT products on Google searches for CRT might be small, we must note that we were not able to control for the timing of product/brand-specific recalls or innovations. Seventh, we focused only on one technology: CRT. As the learning curve on each technology differs, the diffusion of technologies other than CRT may be reflected in a different manner [85].

\section{Conclusion}

This study highlighted the influence of guideline changes on awareness and identified factors that may encourage or discourage the translation of guideline recommendations into technology diffusion. The factors associated with a lack of awareness (as a proxy for technology diffusion) in our case study were: a lack of strong clinical evidence that resulted in the moderate strength of a recommendation, a lack of recognition of any externally published recommendation by the national guideline body (NICE), and the frequent release of guidelines with minor changes targeting small patient groups. At least in our case, in the absence of the UK-based NICE guidelines, the US guidelines received more attention than their non-UK European counterparts, even if the former were released after the latter.

Acknowledgements This study received funding from the European Union Horizon 2020 Research and Innovation Programme under the Marie Skłodowska-Curie Grant agreement no. 721402.

\section{Declarations}

Funding Open Access funding enabled and organized by Projekt DEAL.

Conflict of interest $\mathrm{RV}$ is employed at Abbott for $\mathrm{PhD}$ position created by ITN-IQCE Marie Skłodowska-Curie Grant agreement no. 721402. TS has no conflict of interest to declare.

Data availability statement The datasets generated during and/or analysed during the current study are available from the corresponding author on reasonable request.

Author Contributions RV and TS developed the idea; data extraction and analyses were performed by RV, which were validated by TS; RV wrote the manuscript, which was critically reviewed and edited by TS. Both the authors discussed the results, contributed to final manuscript, and approved the manuscript.

Open Access This article is licensed under a Creative Commons Attribution-NonCommercial 4.0 International License, which permits any non-commercial use, sharing, adaptation, distribution and reproduction 
in any medium or format, as long as you give appropriate credit to the original author(s) and the source, provide a link to the Creative Commons licence, and indicate if changes were made. The images or other third party material in this article are included in the article's Creative Commons licence, unless indicated otherwise in a credit line to the material. If material is not included in the article's Creative Commons licence and your intended use is not permitted by statutory regulation or exceeds the permitted use, you will need to obtain permission directly from the copyright holder. To view a copy of this licence, visit http://creativecommons.org/licenses/by-nc/4.0/.

\section{References}

1. Rogers EM. Diffusion of innovations. 4th ed. New York: Free Press; 2010.

2. Coleman J, Katz E, Menzel H. The diffusion of an innovation among physicians. Sociometry. 1957;20(4):253-70.

3. Greenhalgh T, et al. Diffusion of innovations in service organizations: systematic review and recommendations. Milbank Q. 2004;82(4):581-629.

4. Fleuren M, Wiefferink K, Paulussen T. Determinants of innovation within health care organizations: literature review and Delphi study. Int J Qual Health Care. 2004;16(2):107-23.

5. Meyer AD, Goes JB. Organizational assimilation of innovations: a multilevel contextual analysis. Acad Manag J. 1988;31(4):897-923.

6. WHO. Priority Medical Devices-Background paper 6: Barriers to innovation in the field of medical devices. 2007. https://apps. who.int/iris/handle/10665/70457.

7. Teyhen DS, et al. Leveraging technology: creating and sustaining changes for health. Telemed J E Health. 2014;20(9):835-49.

8. Dirksen CD, Ament AH, Go PMN. Diffusion of six surgical endoscopic procedures in the Netherlands. Stimulating and restraining factors. Health Policy. 1996;37(2):91-104.

9. Lazaro P, Fitch K. The distribution of "big ticket" medical technologies in OECD countries. Organisation for Economic Cooperation and Development. Int J Technol Assess Health Care. 1995;11(3):552-70.

10. Slade EP, Anderson GF. The relationship between per capita income and diffusion of medical technologies. Health Policy. 2001;58(1):1-14

11. Dickson M, Jacobzone S. Pharmaceutical use and expenditure for cardiovascular disease and stroke: a study of 12 OECD countries. OECD health working paper. 1. 2003

12. Oh EH, Imanaka Y, Evans E. Determinants of the diffusion of computed tomography and magnetic resonance imaging. Int $\mathrm{J}$ Technol Assess Health Care. 2005;21(1):73-80.

13. Packer C, Simpson S, Stevens A. International diffusion of new health technologies: a ten-country analysis of six health technologies. Int J Technol Assess Health Care. 2006;22(4):419-28.

14. Gratwohl A, et al. Current trends in hematopoietic stem cell transplantation in Europe. Blood. 2002;100(7):2374.

15. Torbica A, Cappellaro G. Uptake and diffusion of medical technology innovation in Europe: what role for funding and procurement policies? J Med Mark. 2010;10(1):61-9.

16. Mylotte D, et al. Transcatheter aortic valve replacement in Europe: adoption trends and factors influencing device utilization. J Am Coll Cardiol. 2013;62(3):210-9.

17. Cromwell J, Kanak JR. The effects of prospective reimbursement programs on hospital adoption and service sharing. Health Care Financ Rev. 1982;4(2):67-88.

18. Perry S. Diffusion of new technologies: rational and irrational. J Health Care Technol. 1984;1(2):73-88.
19. Romeo AA, Wagner JL, Lee RH. Prospective reimbursement and the diffusion of new technologies in hospitals. J Health Econ. 1984;3(1):1-24

20. McClellan M, Kessler D. A global analysis of technological change in health care: the case of heart attacks. The TECH Investigators. Health Aff (Millwood). 1999;18(3):250-5.

21. Greenberg D, et al. Reimbursement policies, incentives and disincentives to perform laparoscopic surgery in Israel. Health Policy. 2001;56(1):49-63.

22. Cappellaro G, Ghislandi S, Anessi-Pessina E. Diffusion of medical technology: the role of financing. Health Policy. 2011;100(1):51-9.

23. Varabyova Y, et al. The determinants of medical technology adoption in different decisional systems: a systematic literature review. Health Policy. 2017;121(3):230-42.

24. Escarce J. Externalities in hospitals and physician adoption of a new surgical technology: an exploratory analysis. J Health Econ. 1996;15(6):715-34.

25. Callea G, et al. Learning effect and diffusion of innovative medical devices: the case of transcatheter aortic valve implantation in Italy. J Comp Eff Res. 2017;6(4):279-92.

26. Kyratsis Y, Ahmad R, Holmes A. Technology adoption and implementation in organisations: comparative case studies of 12 English NHS Trusts. BMJ Open. 2012;2:e000872. https:// doi.org/10.1136/bmjopen-2012-000872.

27. Sackett DL, et al. Evidence based medicine: what it is and what it isn't. BMJ. 1996;312(7023):71-2.

28. Timmermans S. From autonomy to accountability: the role of clinical practice guidelines in professional power. Perspect Biol Med. 2005;48(4):490-501.

29. Grimshaw J, Eccles M, Tetroe J. Implementing clinical guidelines: current evidence and future implications. J Contin Educ Health Prof. 2004;24(Suppl 1):S31-S3737.

30. Tarricone R, Torbica A, Drummond M. Key recommendations from the MedtecHTA Project. Health Econ. 2017;26(S1):145-52.

31. Kenny T. The nuts and bolts of cardiac resynchronization therapy. Wiley-Blackwell; 2008. pp. 1-218. https://doi.org/10.1002/97804 70751602

32. Jaffe LM, Morin DP. Cardiac resynchronization therapy: history, present status, and future directions. Ochsner J. 2014;14(4):596-607.

33. Bristow MR, et al. Cardiac-resynchronization therapy with or without an implantable defibrillator in advanced chronic heart failure. N Engl J Med. 2004;350(21):2140-50.

34. Cleland JGF, et al. The effect of cardiac resynchronization on morbidity and mortality in heart failure. $\mathrm{N}$ Engl $\mathrm{J}$ Med. 2005;352(15):1539-49.

35. Linde C, Ellenbogen K, McAlister FA. Cardiac resynchronization therapy (CRT): clinical trials, guidelines, and target populations. Heart Rhythm. 2012;9(8):S3-S13.

36. Auricchio A, et al. The pacing therapies for congestive heart failure (PATH-CHF) study: rationale, design, and endpoints of a prospective randomized multicenter study1. Am J Cardiol. 1999;83(5):130-5.

37. EHRA. EHRA webpage. https://www.ehra-whitebook-analysis. com/. Accessed Apr 2018.

38. Farnum, N.R. Quantitative forecasting methods. [2. Dr.] ed. The Duxbury series in statistics and decision sciences. In: Stanton LW, editor. PWS-Kent; 1992.

39. Westbrook JI, Gosling AS, Coiera E. Do clinicians use online evidence to support patient care? A study of 55,000 clinicians. J Am Med Inform Assoc JAMIA. 2004;11(2):113-20.

40. Mikalef P, Kourouthanassis PE, Pateli AG. Online information search behaviour of physicians. Health Inf Libr J. 2017;34(1):58-73. 
41. Steinbrook R. Searching for the right search-reaching the medical literature. N Engl J Med. 2006;354(1):4-7.

42. Google, I. Google Trends Website. https://www.support.googl e.com/trend s/answer/6248105?hl=en\&ref_topic $=6248052$. Accessed July 2018.

43. Scotland, H.I. NICE appraisal considered by Healthcare Improvement Scotland. https://www.healthcareimprovementscotland.org/ our_work/technologies_and_medicines/mta_resources/appra isal_314.aspx?theme=mobile. Accessed Nov 2019.

44. Scotland, H.I. NICE appraisal considered by NHS Quality Improvement Scotland. 2019. https://www.healthcareimprovemen tscotland.org/our_work/technologies_and_medicines/mta_resou rces/appraisal_120.aspx?theme=mobile. Accessed Nov 2019.

45. Swedberg K, et al. Guidelines for the diagnosis and treatment of chronic heart failure: executive summary (update 2005): the task force for the diagnosis and treatment of chronic heart failure of the European Society of Cardiology. Eur Heart J. 2005;26(11):1115-40.

46. Gregoratos $\mathrm{G}$, et al. ACC/AHA/NASPE 2002 guideline update for implantation of cardiac pacemakers and antiarrhythmia devices: summary article. Circulation. 2002;106(16):2145-61.

47. Hunt Sharon A, et al. ACC/AHA 2005 guideline update for the diagnosis and management of chronic heart failure in the adult. Circulation. 2005;112(12):e154-e235.

48. Barnett D, Phillips S, Longson C. Cardiac resynchronisation therapy for the treatment of heart failure: NICE technology appraisal guidance. Heart (British Cardiac Society). 2007;93(9):1134-5.

49. Vardas PE, et al. Guidelines for cardiac pacing and cardiac resynchronization therapy: the task force for cardiac pacing and cardiac resynchronization therapy of the European Society of Cardiology. Developed in collaboration with the European Heart Rhythm Association. Eur Heart J. 2007;28(18):2256-95.

50. Epstein Andrew E, et al. ACC/AHA/HRS 2008 guidelines for device-based therapy of cardiac rhythm abnormalities. Circulation. 2008;117(21):e350-e408.

51. Dickstein K, et al. 2010 focused update of ESC guidelines on device therapy in heart failure: an update of the 2008 ESC guidelines for the diagnosis and treatment of acute and chronic heart failure and the 2007 ESC guidelines for cardiac and resynchronization therapy. Developed with the special contribution of the Heart Failure Association and the European Heart Rhythm Association. EP Europace. 2010;12(11):1526-36.

52. Tracy CM, et al. 2012 ACCF/AHA/HRS focused update incorporated into the ACCF/AHA/HRS 2008 guidelines for device-based therapy of cardiac rhythm abnormalities: a report of the American College of Cardiology Foundation/American Heart Association task force on practice guidelines and the Heart Rhythm Society. J Am Coll Cardiol. 2013;61(3):e6-e75.

53. Boriani $\mathrm{G}$, et al. Cardiac resynchronization therapy: how did consensus guidelines from Europe and the United States evolve in the last 15 years? Int J Cardiol. 2018;261:119-29.

54. (NICE), Implantable cardioverter defibrillators and cardiac resynchronisation therapy for arrhythmias and heart failure (TA314) 2014 04/03/2019]. https://www.nice.org.uk/guidance/ta314/chapt er/1-Guidance. Accessed Apr 2018.

55. Yancy Clyde W, et al. 2013 ACCF/AHA guideline for the management of heart failure. Circulation. 2013;128(16):e240-e327.

56. Brignole M, et al. 2013 ESC Guidelines on cardiac pacing and cardiac resynchronization therapy: The Task Force on cardiac pacing and resynchronization therapy of the European Society of Cardiology (ESC). Developed in collaboration with the European Heart Rhythm Association (EHRA). EP Europace. 2013;15(8):1070-118.

57. Box GEP. Time series analysis forecasting and control. In: Jenkins GM, Reinsel GC, Ljung GM, editors. Wiley series in probability and statistics. 5th ed. New York: Wiley; 2016.
58. Dickey DA, Fuller WA. Distribution of the estimators for autoregressive time-series with a unit root. J Am Stat Assoc. 1979;74(366):427-31.

59. Dickey DA. Stationarity issues in time series models. In: SAS Users Group International 30 proceedings. SAS Global Forum in parternship. SAS, Inc.; 2005.

60. Said SE, Dickey DA. Testing for unit roots in autoregressive-moving average models of unknown order. Biometrika. 1984;71(3):599-607.

61. Phillips PCB, Perron P. Testing for a unit root in time series regression. Biometrika. 1988;75(2):335-46.

62. Kwiatkowski D, et al. Testing the null hypothesis of stationarity against the alternative of a unit root: how sure are we that economic time series have a unit root? J Econometr. 1992;54(1):159-78.

63. Falk M, et al. A first course on time series analysis: examples with SAS. 2005. https://www.statistik.mathematik.uni-wuerzburg.de/ timeseries/.

64. Cazeau S, et al. Effects of multisite biventricular pacing in patients with heart failure and intraventricular conduction delay. N Engl J Med. 2001;344(12):873-80.

65. Gregoratos G, et al. ACC/AHA guidelines for implantation of cardiac pacemakers and antiarrhythmia devices: executive summarya report of the American College of Cardiology/American Heart Association task force on practice guidelines (Committee on Pacemaker Implantation). Circulation. 1998;97(13):1325-35.

66. Moss AJ, et al. Cardiac-resynchronization therapy for the prevention of heart-failure events. N Engl J Med. 2009;361(14):1329-38.

67. Dickstein K, et al. CRT survey II: a European Society of Cardiology survey of cardiac resynchronization therapy in 11088 patients - who is doing what to whom and how? Eur J Heart Fail. 2018;20(6):1039-51.

68. Curtis $\mathrm{AB}$, et al. Biventricular pacing for atrioventricular block and systolic dysfunction. N Engl J Med. 2013;368(17):1585-93.

69. Bogale N, et al. The European CRT Survey: 1 year (9-15 months) follow-up results. Eur J Heart Fail. 2012;14(1):61-73.

70. Muramoto ML, et al. Tobacco cessation skills certification in Arizona: application of a state wide, community based model for diffusion of evidence based practice guidelines. Tob Control. 2000;9(4):408.

71. McCormick L, O'HaraTompkins N. Diffusion of CDC's guidelines to prevent tobacco use and addiction. J Sch Health. 1998;68:43-5.

72. Mold J, et al. Leveraging practice-based research networks to accelerate implementation and diffusion of chronic kidney disease guidelines in primary care practices: a prospective cohort study. Implement Sci IS. 2014;9:169.

73. Lau F, et al. The diffusion of an evidence-based disease guidance system for managing stroke. Int J Med Inform. 1998;51(2):107-16.

74. Van Bockstael V, Sabin N, Crochet B. Impact of guidelines diffusion and information feedback in diabetes management. Assessment of general practitioner support program conducted by the French Mutualite Sociale Agricole (MSA). La Revue du praticien. 2009;59(8 Suppl):13-8.

75. Celentano D, Morlock LL, Malitz FE. Diffusion and adoption of CDC guidelines for the prevention and control of nosocomial infections in US hospitals. Infect Control. 1987;8:415-23.

76. Lia-Hoagberg B, Schaffer M, Strohschein S. Public health nursing practice guidelines: an evalution of dissemination and use. Public Health Nurs. 2002;16(6):397-404.

77. Randolph TC, et al. Utilization of cardiac resynchronization therapy in eligible patients hospitalized for heart failure and its association with patient outcomes. Am Heart J. 2017;189:58.

78. Lund LH, et al. Association between demographic, organizational, clinical, and socio-economic characteristics and underutilization of cardiac resynchronization therapy: results from the Swedish Heart Failure Registry. Eur J Heart Fail. 2017;19(10):1270-9.

79. Sridhar AR, Yarlagadda V, Parasa S, et al. Cardiac resynchronization therapy: US trends and disparities in utilization and outcomes. 
Circ Arrhythm Electrophysiol. 2016;9(3):e003108. https://doi. org/10.1161/CIRCEP.115.003108.

80. Jonathan $\mathrm{P}$, et al. Use of cardiac resynchronization therapy in patients hospitalized with heart failure. Circulation. 2008;118(9):933.

81. Mohammadreza Hsseini S, et al. Utilization and in-hospital complications of cardiac resynchronization therapy: trends in the United States from 2003 to 2013. Eur Heart J. 2017;38:2122-8.

82. Lyons KJ, et al. Impact of current versus previous cardiac resynchronization therapy guidelines on the proportion of patients with heart failure eligible for therapy. JACC Heart Fail. 2017;5(5):388-92.
83. Dubois RW, Dean BB. Evolution of clinical practice guidelines: evidence supporting expanded use of medicines. Dis Manag. 2006;9(4):210-23.

84. Normand $\mathrm{C}$, et al. Indications for cardiac resynchronization therapy: a comparison of the major international guidelines. JACC Heart Fail. 2018;6(4):308-16.

85. Govindarajulu US, Stillo M, Goldfarb D, Matheny ME, Resnic FS. Learning curve estimation in medical devices and procedures: hierarchical modeling. Statist Med. 2017;36:2764-85. https://doi. org/10.1002/sim.7309. 